\title{
ADVANCES IN THE MESOSCALE APPROACH TO STRUCTURE-SENSITIVE PROPERTIES RELATIONS IN POLYCRYSTALLINE MATERIALS
}

\author{
B.L. ADAMS \\ Department of Materials Science and Engineering, Carnegie Mellon \\ University, 5000 Forbes Avenue, Pittsburgh, PA 15213-3890, USA
}

(Received in final form 28 September 1997)

\begin{abstract}
New methods have recently been conceived for extracting certain intrinsic properties of grain boundaries. Described here is a novel approach to extracting the excess free energy and mobility functions over large components of the entire fundamental space of grain boundary types. The theoretical approach constitutes a new form of inverse problems in materials science, and demands geometrical and orientational characterizations of very large numbers of triple junctions. The experimental approach demands new levels of automation of orientation imaging microscopy, and calibrated serial sectioning to overcome the opacity problem in electron diffraction. The main threads of the approach (theoretical, numerical and experimental) are described in this paper.
\end{abstract}

Keywords: Polycrystalline aggregates; Structure-sensitive properties; Grain boundaries; Excess free energy; Mobility; Statistical methods

\section{INTRODUCTION}

For more than sixty years, physical properties have been classified into two broad categories: structure-insensitive vs structure-sensitive (Smekal, 1933; Seeger, 1958). The distinction between the two is never fully realized in any material or for any particular property, but it has remained a useful distinction. What is meant is that some properties are observed to be far more sensitive to the details of internal structure than others. (Internal structure refers here to any deviation from the idealized strain and defect free crystalline phases, including the grain 
boundaries associated with polycrystallinity.) Examples of structureinsensitive properties include elastic stiffness and electrical or thermal conductivity in metals and ceramics (but not semiconductors) over a broad range of temperatures. Examples of structure-sensitive properties include intergranular embrittlement and stress corrosion cracking, where grain boundary type is known to be important. (Gottstein and Schwarzer (1992) and Lejcek and Hofmann (1995) have given a much broader perspective on structure-sensitive properties than is possible here.)

In this paper we detail theoretical and experimental progress towards the determination of certain important intrinsic properties of grain boundaries in polycrystalline materials. The main examples are the excess free energy and the mobility functions. These functions have the form

$$
f: \Gamma \backslash S O(3) / \Gamma \times S^{2} \rightarrow \Re^{+},
$$

where $\Gamma \backslash S O(3) / \Gamma \times S^{2}$ denotes the five-parameter fundamental space of grain boundary types (specified by crystal misorientation and boundary inclination) and $\Re^{+}$denotes the set of non-negative real numbers. Although the focus on free energy and mobility is somewhat removed from the problem of macroscopic structure-sensitive properties, it is probable that an understanding of such intrinsic properties must precede a deeper understanding of macroscopic properties. Also, it is wellunderstood that the free energy and mobility of grain boundaries substantially determine the evolution of texture and microstructure during coarsening through a complex interplay of the thermodynamic driving forces and kinetic responses which determine the motions of grain boundaries. Thus, progress towards understanding and control of microstructural evolution depends upon gaining a systematic understanding of how the free energy and mobility vary as a function of grain boundary type and the relevant thermodynamic variables.

We proceed with a brief review of theoretical progress which has been achieved towards the determination of the free energy and mobility functions over the full fundamental space of grain boundary types. (More details can be found in the recent paper of Adams et al., 1997.) The subsequent section describes progress in the development of advanced orientation imaging methods which are capable of providing 
the required mesostructural data. The final section will provide a summary and conclusions of the work.

\section{DETERMINATION OF THE RELATIVE FREE ENERGY AND MOBILITY FUNCTIONS FROM THE MESOSTRUCTURE}

This section describes a method for extracting, from measurements of the mesostructure of polycrystals, the relative excess free energy and the relative mobility of the grain boundaries as functions of the crystallographic type (five degrees of freedom) and relevant thermodynamic variables. The method requires the simultaneous measurement of both the geometry and the crystallography of a large number of grain boundary intersections; the crystallographic information may be obtained by an extension of orientation imaging microscopy (OIM) as will be described in the next section. The intersections are assumed to be triple junctions (three intersecting boundaries). The energies and mobilities are obtained as ratios to respective standards that must be determined independently.

The determination of grain boundary energies follows from the assumption of local equilibrium at the triple junctions; each triple junction is assumed to obey Herring's (1952) equilibrium equations. Herring's relations can be written as

$$
\sum_{j=1}^{3}\left\{\sigma_{j} \hat{b}_{j}+\left(\partial \sigma_{j} / \partial \phi_{j}\right) \hat{n}_{j}\right\}=0
$$

where $\sigma_{j}$ is the excess free energy of the $j$ th boundary, $\hat{n}_{j}$ is the unit boundary normal of the $j$ th boundary, $\hat{b}_{j}$ is a unit vector lying in the $j$ th boundary and perpendicular to the triple line $\hat{l}=\hat{n}_{j} \otimes \hat{b}_{j}$ which is common to all three adjacent boundaries, and $\phi_{j}$ is defined to be the right-handed angle of rotation about $\hat{l}$ of the $j$ th boundary from a reference direction. If a boundary lies in an energy cusp orientation, special care must be taken in defining the corresponding torque term in Eq. (2) (Herring, 1952; Mullins, 1963). Equation (2), when considered for a large set of triple junctions, provides a system of equations which can be solved to determine the free energy function. 
Grain boundary velocities at a triple junction are related since the motion of any two of the intersecting boundaries defines a new locus of intersection to which the third boundary must move if the triple junction is to remain intact. The equation connecting the velocities becomes an equation connecting the mobilities when the driving forces are specified; under ordinary circumstances, the latter require knowledge of the local curvature and energy function of each grain boundary at the triple junction.

Let $\mathbf{V}$ be the velocity of the triple junction in the direction normal to the triple junction line, and $v_{i}$ be the velocity of the $i$ th attached grain boundary in the direction of its normal. Then if the triple junction remains intact, the relation

$$
v_{i}=\mathbf{V} \cdot \hat{n}_{i}
$$

must be satisfied, where $\hat{n}_{i}$ is the corresponding normal; here the grain boundary normals are chosen in the same sense around the triple junction as shown in Fig. 1. The normals are linearly dependent since any one can be expressed in terms of the other two. Use of cross products establishes that the relation among them is

$$
\sum_{i} \sin \left(\chi_{i}\right) \hat{n}_{i}=0 .
$$

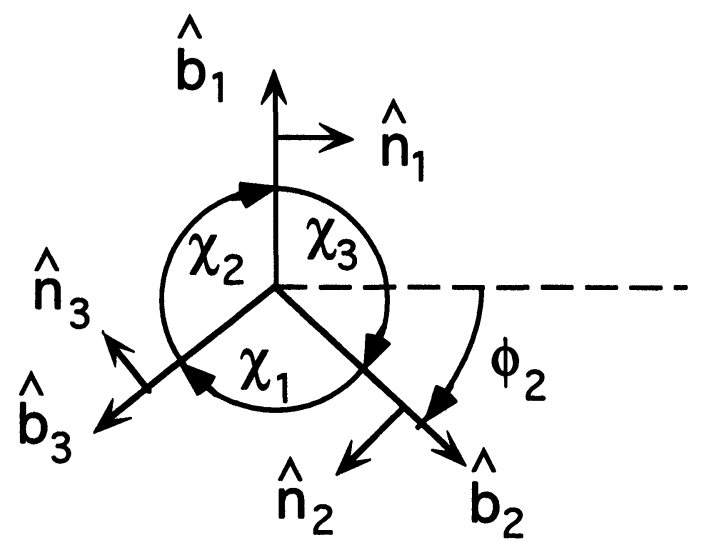

FIGURE 1 Definition of geometric parameters associated with boundaries adjoining the triple junction. Each boundary is associated with a (unit) normal, $n$, a (unit) direction, $b$, an inclination, $\phi$, and a dihedral angle, $\chi$. Only one inclination angle $\left(\phi_{2}\right)$ is labeled for clarity. 
Therefore, multiplying Eq. (3) by $\sin \left(\chi_{i}\right)$ and summing on $i$ gives

$$
\sum_{i} \sin \left(\chi_{i}\right) v_{i}=0
$$

as the relation among the normal velocities at the triple junction. If we assume that these velocities are given by $v_{i}=m_{i} f_{i}$, where $m_{i}$ is the mobility and $f_{i}$ is the local driving force, then

$$
\sum_{i}\left\{\sin \left(\chi_{i}\right) f_{i}\right\} m_{i}=0
$$

When the coefficients of the $m_{i}$ (i.e. the products $\left.\sin \left(\chi_{i}\right) f_{i}\right)$ are known, then Eq. (6) connects the unknown mobilities. But the coefficients are determined by the angles of intersection $\chi_{i}$ and by the driving forces given by the Herring (1952) formula,

$$
f=\left(\sigma+\sigma_{\theta_{1} \theta_{1}}\right) \kappa_{1}+\left(\sigma+\sigma_{\theta_{2} \theta_{2}}\right) \kappa_{2},
$$

in which $\kappa_{1}$ and $\kappa_{2}$ are the principal curvatures, and $\sigma_{\theta_{1} \theta_{1}}$ and $\sigma_{\theta_{2} \theta_{2}}$ are angular derivatives of $\sigma$ along the principal curves; hence the $f_{i}$ are determined by the curvatures and energy functions of the boundaries at the triple junctions. Given these quantities, the coefficients of Eq. (6) are determined and the equation represents one relation among the unknown mobilities for each triple junction. From a large number of triple junctions, numerous relations of the type shown in Eq. (6) can be constructed to form a basis for extracting the mobility function.

The discussion is limited to the method of extraction of the free energy function from triple junction measurements. The same procedure can be used to extract the mobility function. The only difference is that two independent relations among energies are available at each triple junction whereas only one relation among mobilities is available. The method is statistical and its implementation is multiscale.

Discretize the fundamental space into $N$ elements $\delta \omega_{i} \subset \Gamma \backslash S O(3) /$ $\Gamma \times S^{2}$ such that

$$
\begin{aligned}
& \delta \omega_{i} \cap \delta \omega_{j}=\varnothing \quad \text { for } i \neq j, \\
& \cup \delta \omega_{i}=\Gamma \backslash S O(3) / \Gamma \times S^{2} .
\end{aligned}
$$


Since each grain boundary can be characterized by misorientation and inclination parameters falling into one of the $N$ elements of the fundamental zone, we shall express the free energy of any particular boundary as

$$
\sigma(\omega) \equiv \sigma_{i} \text { when } \omega \in \delta \omega_{i}
$$

Also, tabulate the Herring's equations for each triple junction available in the data set according to a discrete assignment of free energies for each grain boundary of the triple junction. Since only two of the three Herring's relations (Eq. (2)) are independent, such an assignment involves only two free energies from the fundamental zone, and therefore a pair of the form $\left(\sigma_{i}, \sigma_{j}\right)$. These free energies are associated with a pair of discrete types $\left(\delta \omega_{i}, \delta \omega_{j}\right)$. Establish $\left(\begin{array}{l}N \\ 2\end{array}\right)=N(N-1) / 2$ urns for the pairs $\left(\delta \omega_{i}, \delta \omega_{j}\right)$, and place each equation into its respective urn, imposing the statistical hypothesis that each such placement is an independent trial of identically distributed random pairs. We now exploit the Law of Large Numbers which tells us that the running average of the coefficients is normally distributed. This then permits us to average the equations in each urn to obtain a set of $\left(\begin{array}{l}N \\ 2\end{array}\right)$ averaged equations. The objective of this averaging (or coarse graining) procedure is to recast the problem in a robust form suitable for solution. The entire system of Herring's relations can then be incorporated in an equation of the form

$$
\sum_{1}^{N} A_{i j} \sigma_{j}=b_{i}, \quad i=1, \ldots, N(N-1) / 2,
$$

where the matrix of coefficients, $A_{i j}$, then contains the averaged relations between energies, $\sigma_{j}$ is a vector of the energy function to be determined and $b_{i}$ is a zero vector.

It is presumed that $N$ is large, e.g., and therefore Eq. (10) still contains a large number of equations for numerical resolution. Thus it is useful to introduce a multiscale iterative method. The approach begins with a small $N$ (coarse partitioning of the fundamental space) and solves the small system for a trial energy, say $\sigma^{N} . N$ is then increased to $2 N$, by splitting each element of the partition into two pieces. The data set is then resorted and averaged, and resolution at level $2 N$ is initiated 


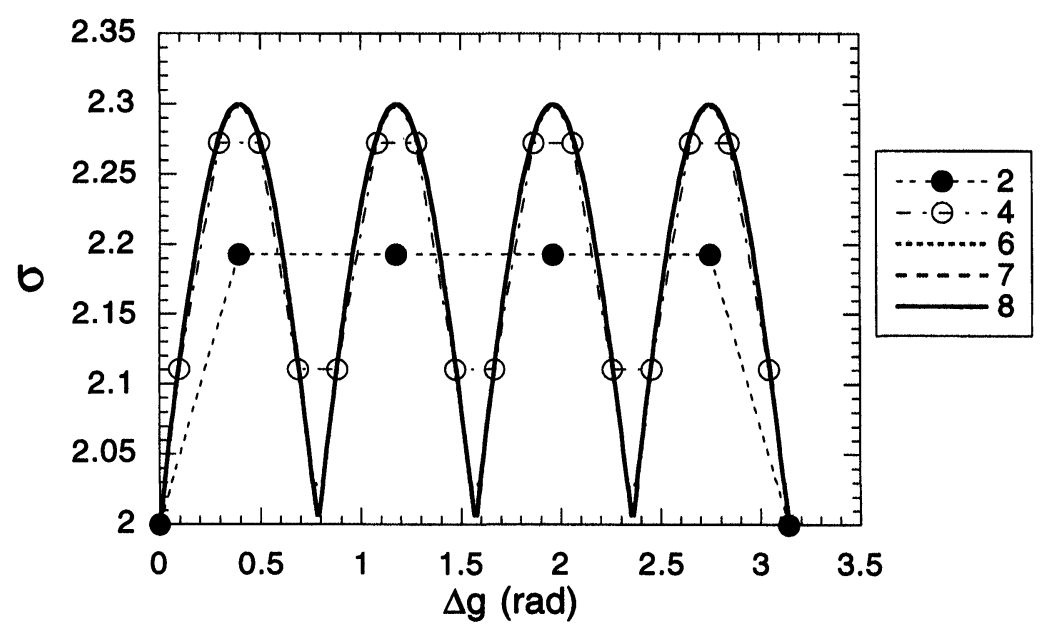

FIGURE 2 Reconstruction of the free energy function, $\sigma$, based on a simulated set of triple junction angles derived from a trigonometric function, showing five of the eight successive orders of approximation, beginning with $N=2$ and ending with $N=256$. The number of points is two raised to the power of the order. Orders 2 $(N=4)$ and $4(N=4)$ are shown in filled and unfilled circles respectively, to emphasize the result of the early stages of approximation, whereas stages 6,7 and $8(N=64$, 128 , and 256) are indistinguishable on this plot, owing to the rapid convergence on the trial function.

with the solution $\sigma^{N}$ of the previous level. It turns out that by adapting a Kaczmarz method, the sparseness of the coefficient matrix is preserved and a solution found rapidly (Adams et al., 1997). An elementary example of this reconstruction is shown in Fig. 2.

The approach for extracting the mobility function would follow on identical lines, except that Eqs. (6) and (7) are used instead of Herring's relations. It should be apparent that the energy function must be known, however, before the mobilities can be extracted. Only the relative energies or mobilities are extracted, and therefore scaling these functions will require additional experimental data not available from the measurements of mesostructure.

\section{DEVELOPMENT OF THE EXPERIMENTAL METHODS}

That the (relative) free energy and mobility functions can be determined, in principle, from measurements of grain boundary type and 
geometry for large numbers of triple junctions is rather remarkable. The approach outlined in the previous section constitutes a new class of inverse problems in materials science. What is required for the freeenergy function is the measurement of the type of boundaries (lattice misorientation and inclination) and their interconnecting dihedral angles for each of a vast number of triple junctions. What is additionally required for the extraction of the mobility function, as can be seen from Eq. (7), is the measurement of the principal curvatures of each boundary at the root of the junction.

The actual number of triple junctions which must be characterized is not known since it is not known whether the complete fundamental space, $\Gamma \backslash S O(3) / \Gamma \times S^{2}$, is fully occupied in nature. In an approximate way, however, the number of required measurements can be estimated from the number of tiles required in partitioning the fundamental space. This number will be a function of the angular resolution in each of the five angular variables in the space, and from the order of the lattice symmetry subgroup $\Gamma$. If we assume that each of the five angular variables of lattice misorientation and inclination are required to the same accuracy, say $\alpha$, then for the most highly symmetrical case of single-phase cubic materials with symmetry subgroup $\Gamma=O_{h}$, the number of tiles, $N$, can be estimated to be (Adams, 1997):

$$
N \cong \frac{\pi^{3}}{144 \alpha^{5}}
$$

Thus, at an angular resolution of $8^{\circ}, \sim 4,000$ grain boundary types occur in the fundamental zone. When $\alpha=4^{\circ}$ this number has increased to $\sim 130,000$, at $2^{\circ}$ it is $\sim 4,155,000$, and at $1^{\circ}$ it is $\sim 133,000,000$. When the statistical aspects of the "goodness of fit" of the discrete function are considered it is evident that a multiple of $N$ may be required to adequately resolve the free energy and mobility functions. The size of these numbers demands new experimental approaches, beyond what has been considered in the past.

A second experimental problem is evident from the requirements. The electron-opacity of most polycrystalline materials is a very old experimental problem that must be confronted in this case. Electron probe methods which obtain electron backscattering diffraction patterns (EBSPs), can now be rapidly indexed without human 
intervention, and thus may provide a basis for the determination of very large numbers of grain boundary misorientations. However, since penetration depths of only about $20 \mathrm{~nm}$ occur, EBSPs are formed very near the surface and they are not suitable for determining both parameters of the grain boundary inclination. The inclination in the section plane is accessible, but the tilt of the boundary away from the section plane normal is not observable. For this determination other approaches must be considered, such as (destructive) serial sectioning.

In that which follows a brief description is given of the approach which is being taken at Carnegie Mellon University to obtain vast quantities of experimental data on the mesostructural parameters of triple junctions, as required to solve for the free energy and mobility functions. The experimental system is still in development.

\section{Advanced Orientation Imaging Microscopy}

Ordinary methods of OIM are not suitable for the simultaneous rapid determination of lattice orientation and the geometry of triple junctions. In the usual method (Adams et al., 1993) the electron beam is used in a spot mode to scan over a systematic array of points on polished surfaces. Orientations are obtained by indexing algorithms at each point of the array, and a graphical picture of the underlying microstructure is obtained by comparing the orientations of neighboring points on the array. Graphical elements are introduced in the computer image when orientation differences between neighboring points suggest the presence of a grain boundary. The resolution of the geometry of the grain boundaries and triple junctions is thus determined by the scale of the scanning array. The number of points at which EBSPs must be obtained increases as the inverse of the square of the mesh size of the array. Since it is desirable to resolve dihedral angles to $\sim 1^{\circ}$, the mesh size must be of the order of $D / 360$ ( $D$ is the grain size) in well annealed materials. Estimating the number of triple junctions in a section to be $2 A / D^{2}$ ( $A$ is the measurement area), it is evident that the number of EBSPs required to adequately obtain the geometry of triple junctions using the conventional method of OIM is a multiple of approximately 64,800 times the number of triple junctions which need to be determined. When it is envisioned that millions of triple junctions must be characterized, and when it is considered that sufficient 
signal-to-noise ratios in EBSPs cannot be obtained in less than $\sim 0.1 \mathrm{~s}$, it becomes very evident that a conventional OIM is not suitable for the problem at hand.

The CMU group is developing a variant of OIM which will be referred here to advanced orientation imaging microscopy (AOIM). The central idea is to use other methods of grain boundary contrast, such as backscattering or secondary electron contrast, to quickly form an image of the geometry of grain boundaries and triple junctions over a field of view which contains a number of triple junctions. An example of such an image, obtained by a solid state backscattering detector, is shown in Fig. 3(a). Using conventional edge detection methods and

(a)

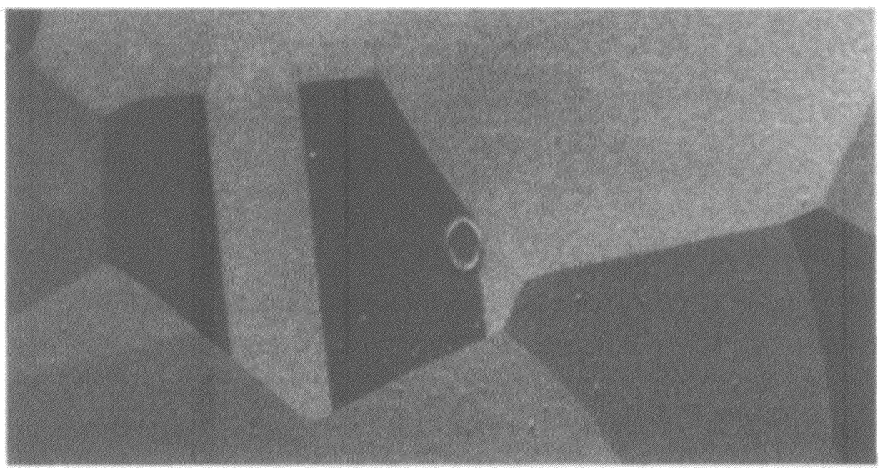

(b)

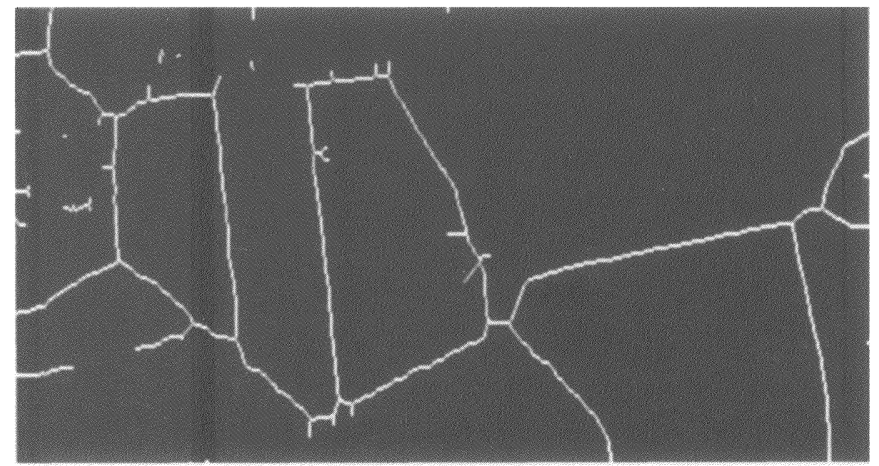

FIGURES 3(a) and (b) 
(c)

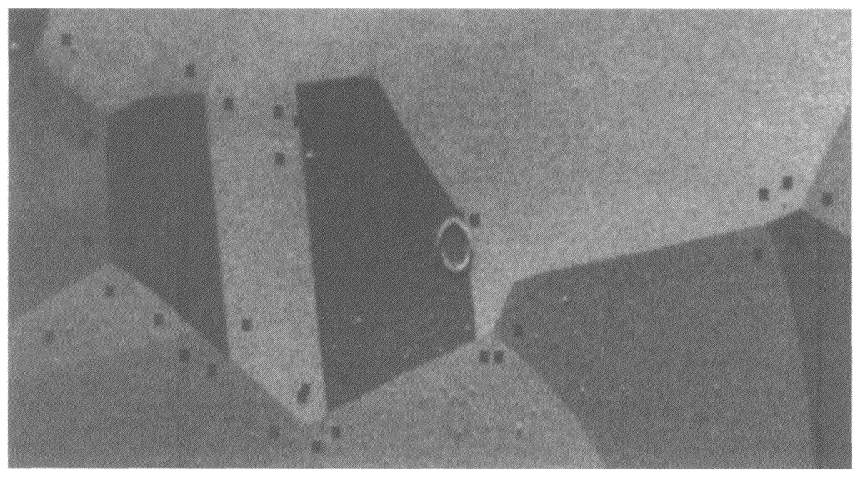

(d)

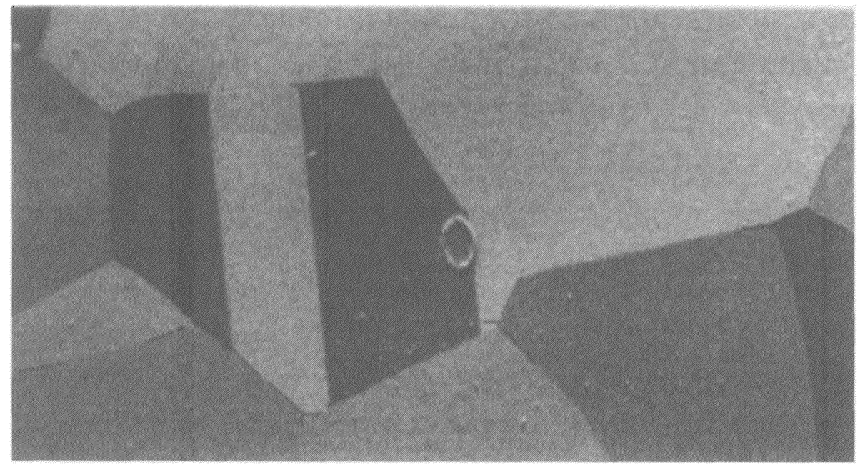

FIGURES 3(c) and (d)

FIGURE 3 Sequence illustrating AOIM: (a) typical backscattering contrast image; (b) image obtained by edge detection and skeletonization; (c) locations of points determined for positioning of electron beam for orientation determination; (d) estimation of (apparent) dihedral angles from skeletonized image.

further processing of the image, a skeletonized image of the grain boundary geometry is obtained. An example is shown in Fig. 3(b). From the geometry of the skeletonized representation an algorithm has been constructed which locates the triple junctions and determines the locations of selected points (three near each triple junction) where the electron probe can be placed in order to determine the lattice 
orientations for the adjacent grains. The results are shown in Fig. 3(c). The digital microscope is then changed from scanning to spot mode, the beam is directed to each required point, an EBSP is obtained and analyzed for each such point, and thus, complete data for several triple junctions are obtained. From the skeletonized image the (apparent) dihedral angles and in-plane curvatures of the grain boundaries can be directly obtained. Figure 3(d) illustrates this by superimposing tangent lines to each participating grain boundary. This process would be repeated many times until sufficient data is obtained. The number of EBSP measurements required is estimated to be reduced from conventional OIM by a factor of $\sim 2 \times 10^{4}$ at the required levels of geometrical resolution. On the basis of the current physical, experimental and computational limitations, we have estimated that it may be feasible to characterize as many as 50,000 triple junctions per hour. Thus, data sets of sufficient size may be achieved over the span of several days time when the system is completely automated. The stability of the current generation of electron microscopes is sufficient to contemplate such long experimental campaigns.

\section{Issues Related to Calibrated Serial Sectioning}

Modern (abrasive) polishing techniques, developed in the optical industries, can be applied to the problem of serial sectioning of polycrystalline samples. When the samples are hard and brittle, such as ceramic polycrystals, the polishing procedures established for optical components achieve similar results. The main additional requirement is the problem of quantifying removal rates which facilitate sufficient control of sectioning. Control of parallelism on continuous polishing systems is not expected to be a problem if reasonable care is taken in the process. The situation with soft, ductile materials, such as pure metals, is rather poorly understood by comparison. Since electropolishing is usually required after (abrasive) polishing, one must account for the additional removals that occur in electropolishing in establishing sectioning procedures. As with much of metallographic practice, calibrated serial sectioning of ductile metals is more art than science.

Once sectioning procedures have been established, it is necessary to obtain a registry of data obtained on subsequent sections. Previous work (cf. Pan et al., 1996) has employed microhardness indentations, 
which are partially removed by serial polishing, to establish registry between adjacent sections. Estimates of the thickness of the removed layer are obtained from a knowledge of the geometry of the indenter. Errors occur mainly from polishing and electropolishing distortions of the geometry of the indent. With respect to the use of indentation marks to be used for the purpose of spatial registry, the aforementioned distortions also tend to degrade the registry process. When the mesostructure of the material can reasonably be assumed to exhibit statistical homogeneity in the distribution of triple-line orientations, and when each triple line can be positively identified by its crystallographic type, then the use of the triple lines themselves as an extensive system of (natural) internal markers is an attractive alternative. This approach is now being investigated in our center.

The matter of establishing registry between discrete data sets is an old problem in the literature, investigated by MacKenzie (1957), McLachlan (1972; 1982), Kabsch (1976; 1978), Mackay (1977), KenKnight (1984) and Diamond (1988; 1990). The full three dimensional problem involves two sets of points in Euclidean space. In the approach we have taken, these points are the triple points which occur in section planes. One set of points associates with the triple points in a specific section, and the second set with the associated triple points in an adjacent section. It is assumed that a one-to-one correspondence can be achieved between many (but not all) elements of these two point sets. Let the coordinates of the $n$th triple point in the first section be $x_{n}^{i}$, and the coordinates for the corresponding triple point in the adjacent section $y_{n}^{i}$. The problem is then to find a translation $\mathbf{y}_{0}$ and a rotation $R$ which will transform the points of the second set to the locations as close as possible to those of the corresponding points of the first set. Formally, we wish to minimize the residue

$$
d=\sum_{n} \sum_{i=1}^{3} \sum_{j=1}^{3} \sum_{k=1}^{3} w_{n}\left(R_{i j}\left(y_{n}^{j}-y_{0}^{j}\right)-x_{n}^{i}\right)\left(R_{i k}\left(y_{n}^{k}-y_{0}^{k}\right)-x_{n}^{i}\right),
$$

where the symbol $w_{n}$ denotes the weight $\left(w_{n} \geq 0\right)$ associated with the $n$th pair of points, normalized such that $\sum_{n} w_{n}=1$. Standard approaches can be taken to solve the problem for $R$ and $\mathbf{y}_{0}$, including the method of Lagrange multipliers. Further details are not given here. 
Two kinds of registry are required by the approach we have taken: first, in connection with AOIM it is necessary to establish registry between adjacent backscattering contrast images lying in a single section plane; the second is the registry between adjacent section planes. The first registry is completed before the second is attempted. In order to facilitate registry between adjacent contrast images it is necessary to provide overlapping data sets. Registry between adjacent sections makes use of the assumption that the distribution of triple line orientations is isotropic (or at a minimum exhibits planar isotropy about the section plane normal direction). Registry is achieved by minimizing the residue in Eq. (12) under the assumption that both the sets of points lie in the same plane. The estimated coordinates are then obtained by adding to the translated and rotated set of points, a vector of length equal to the thickness removed by serial sectioning, and of direction parallel to the section plane normal.

\section{Retrieving Geometrical Parameters from Sectioning Data}

It is evident from relations (2), (6) and (7) that analysis for the free energy and mobility functions requires a determination of the three dihedral angles and six principal curvatures (two for each grain boundary) associated with each triple junction. These geometrical parameters must be determined from data obtained on a sequence of section planes.

Determination of the (true) dihedral angles, $\chi_{1}, \chi_{2}, \chi_{3}$, from the sectioning data is a simple matter when the direction of the triple line, $\hat{l}$, has been determined by the registry procedure described in the previous procedure. Assuming that $\hat{l}$ has been determined and that the apparent dihedral angles (those observed for any particular triple junction in the section plane) have been measured, the true dihedral angle is obtained from the expression

$$
\cos \chi_{t}=\frac{\cos \chi_{a}-\cos \alpha_{1} \cos \alpha_{2}}{\sin \alpha_{1} \sin \alpha_{2}}
$$

Here $\chi_{t}$, is the true dihedral angle, $\chi_{a}$ is the apparent dihedral angle in the section plane, and $\alpha_{1}$ and $\alpha_{2}$ are the angles subtended between $\hat{l}$ and the vectors formed by the intersection of the section plane and the two 
grain boundaries in question. A more complex, but equivalent, expression was given earlier by Harker and Parker (1945).

The matter of extracting the curvatures of grain boundaries from sectioning data is a topic closely related to the general question of fitting two-dimensional surfaces through data sets. An extensive literature for the subject is available. Quadrics (Chernuschi-Frias, 1984; Bolle and Vemuri, 1989), superquadrics (Solina and Bajcsy, 1990), hyperquadrics (Hanson, 1988; Cohen and Cohen, 1994), higher-order algebraic surfaces (Taubin, 1991), splines (Flynn and Jain, 1989) and Monge patches (Renka, 1996) are among the types of surfaces that have been considered in the literature. Methods for extracting the curvatures have been considered by Hilton et al. (1995).

Further discussion of curvature extraction is beyond the scope of this paper. However, the fundamental issue in such determinations can be mentioned. It is that the quality of estimation is expected to be strongly affected by the disparate degrees of absolute precision with which the coordinates of measured data points can be measured. For the two coordinates which lie in the section plane, rather high precision can be achieved; for the third coordinate, the degree of precision is directly related to uncertainties in measuring the amount of material removed in the serial sectioning program. Thus, the third coordinate of surface points will always be measured with less absolute precision than the other two.

\section{SUMMARY AND CONCLUSIONS}

By way of summary, a new approach to structure-sensitive properties has been presented in this paper. The approach focuses upon determining certain key intrinsic properties of grain boundaries. Of central interest are the excess free energy and mobility functions. The theoretical, numerical and experimental challenges were discussed. The mathematical approach requires the characterization of the crystallographic type and the geometry of a vast number of (equilibrated) triple junctions. It was shown how Herring's relation (Eq. (2)) can be solved, using a new multiscale statistical approach, to obtain the functions of interest. This is not a trivial problem because, in general, the equation for any given triple junction connects 
the free energies (and their derivatives) at widely separated values of the independent variables. Straightforward consecutive solution of these equations can result in large instabilities. The new method obviates this problem.

The relative mobilities of the boundaries meeting at a triple junction were shown to satisfy an equation of constraint, assuming that the local grain boundary velocity is proportional to the local thermodynamic driving force (i.e. curvature motion). A large number of such equations, one for each triple junction, may be treated by the same method used in the energy case resulting in the relative mobility as a function of the grain boundary type (and thermodynamic variables).

It is remarkable that (relative) free energies and mobilities may be inferred from the microstructure at a given instant; absolute free energies and mobilities (requiring evolution data) are required only to establish the absolute free energy and mobility of standards (e.g., comprising the motion of a particular grain boundary under a known driving force).

The experimental requirements were then discussed. It is emphasized that the hardware and software necessary to obtain data of sufficient quality and quantity is still being developed. Three aspects of the experimental program were described.

The first has to do with obtaining sufficient numbers of data on triple junctions, and expanding the capabilities of OIM. It is clear that conventional OIM is not suitable without substantial modifications. The main challenges include increasing the rate at which EBSPs can be reliably indexed, and the problem of simultaneously obtaining geometrical information on triple junctions along with the orientational information. A particular approach, here called AOIM, was described; the main advance is that of a (spatial) coupling of backscattering contrast imaging with the indexing capabilities of OIM. It is now apparent that such advances may provide data of sufficient quality for millions of triple junctions in reasonable experimental times.

The second experimental challenge has to do with obtaining a registry between neighboring data sets. This is critical to AOIM, since contrast images must be performed over areas which are relatively limited in area (numbers of triple points in the section area). It is also critical to overcoming the limitations of electron opacity, and the need to study the third dimension of sample space by calibrated serial sectioning. The approach being developed at CMU was briefly discussed. 
Finally, the problem of extracting the specific geometrical data required for each triple junction (dihedral angles and principal curvatures) was discussed. The available literature on relevant related problems is extensive; here a brief review of the available approaches was provided.

\section{Acknowledgments}

The author wishes to acknowledge the numerous contributions to this work by his colleagues and students affiliated with the Mesoscale Interface Mapping Project at Carnegie Mellon University. These include W.W. Mullins, D. Kinderlehrer, A.D. Rollett, C.L. Bauer, S. Ta'asan, D. Casasent, A. Morawiec, C.-T. Wu and A. Talukder. Relations (12) and (13) were derived by A. Morawiec. The new method for extracting mobilities was first developed by W.W. Mullins. The multiscale modeling approach has been developed by D. Kinderlehrer and S. Ta'asan. Figure 3 was kindly provided by C.-T. Wu and A. Talukder. This work was supported primarily by the MRSEC Program of the National Science Foundation under Award Number DMR-9632556.

\section{References}

Adams, B.L., Wright, S.I. and Kunze, K. (1993) Metall. Trans., 24A, 819.

Adams, B.L. (1997) Ultramicroscopy, 67, 11-17.

Adams, B.L., Kinderlehrer, D., Mullins, W.W., Rollett, A.D. and Ta'asan, S. (1997) Scripta Mater. (submitted).

Bolle, R.M. and Vemuri, B.C. (1989) IBMC 14557.

Chernuschi-Frias, B. (1984) Orientation and location parameter estimation of quadratic surfaces in 3-D from sequence of images, Ph.D. Dissertation, Brown University, Div. of Engineering, Providence, RI.

Cohen, I. and Cohen, L.D. (1994) Proc. 12th Int. Conf. Pattern Recognition, Los Alamos, CA, USA, p. 403.

Diamond, R. (1988) Acta Cryst. A, 44, 211-216.

Diamond, R. (1990) Acta Cryst. A, 46, 423.

Flynn, P.J. and Jain, A.K. (1989) Proc. IEEE Computer Society Conf. on Computer Vision and Pattern Recognition, IEEE Computer Society Press, Washington, D.C., USA, p. 110.

Gottstein, G. and Schwarzer, F. (1992) Mat. Sci. Forum, 94-96, 187-208.

Hanson, A.J. (1988) Comp. Vision, Graphics and Image Processing, 44, 191.

Harker, D. and Parker, E. (1945) Trans. Amer. Soc. Metals, 34, 156.

Herring, C. (1952) in Structure and Properties of Solid Surfaces, R. Gomer and C.S. Smith, eds, University of Chicago Press.

Hilton, A., Illingworth, J. and Windeatt, T. (1995) Pattern Recognition, 28, 1201.

Kabsch, W. (1976) Acta Cryst. A, 32, 922-923. 
Kabsch, W. (1978) Acta Cryst. A, 34, 827-828.

KenKnight, C.E. (1984) Acta Cryst. A, 40, 708-712.

Lejcek, P. and Hofmann, S. (1995) CRC Critical Reviews in Solid State and Materials Science, $\mathbf{2 0}, 1$.

Mackay, A.L. (1977) Acta Cryst. A, 33, 212-215.

MacKenzie, J.K. (1957) Acta Cryst., 10, 61-62.

McLachlan, A.D. (1972) Acta Cryst. A, 28, 656-657.

McLachlan, A.D. (1982) Acta Cryst. A, 38, 871-873.

Mullins, W.W. (1963) in Metal Surfaces: Structure, Energetics and Kinetics, W.D. Robertson and N.A. Gjostein, eds, The American Society for Metals, Ohio, p. 17.

Pan, Y., Adams, B.L., Olson, T. and Panayotou, N. (1996) Acta Mater., 44, 4685-4695. Renka, R.J. (1996) ACM Transactions on Mathematical Software, 22, 9.

Seeger, A. (1958) in Handbuch der Physik, Vol. 7, S. Flügge (ed.), Springer-Verlag, Berlin. Smekal, A. (1933) in Handbuch der Physik, Vol. 2, Springer-Verlag, Berlin.

Solina, F. and Bajcsy, R. (1990) IEEE Trans. Patt. Anal. Mach. Intell., 12, 131.

Taubin, G. (1991) IEEE Trans. Patt. Anal. Mach. Intell., 13, 1115. 